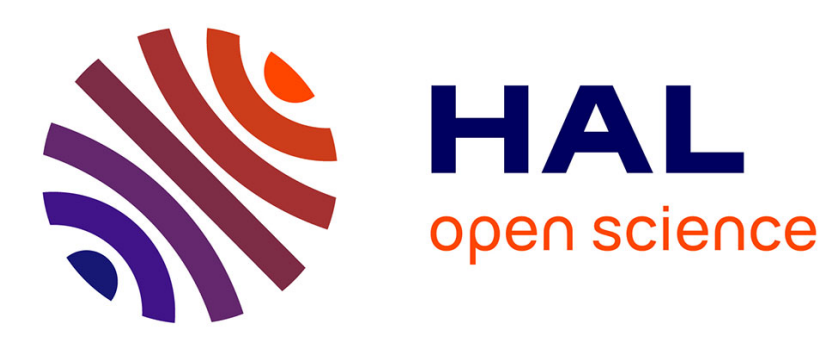

\title{
Learning Users' and Personality-Gender Preferences in Close Human-Robot Interaction
}

\author{
Arturo Cruz-Maya, Adriana Tapus
}

\section{To cite this version:}

Arturo Cruz-Maya, Adriana Tapus. Learning Users' and Personality-Gender Preferences in Close Human-Robot Interaction. The 26th IEEE International Conference on Robot and Human Interactive Communication, Aug 2017, Lisbonne, Portugal. 10.1109/ROMAN.2017.8172393 . hal-01698359

\section{HAL Id: hal-01698359 \\ https://hal.science/hal-01698359}

Submitted on 1 Feb 2018

HAL is a multi-disciplinary open access archive for the deposit and dissemination of scientific research documents, whether they are published or not. The documents may come from teaching and research institutions in France or abroad, or from public or private research centers.
L'archive ouverte pluridisciplinaire HAL, est destinée au dépôt et à la diffusion de documents scientifiques de niveau recherche, publiés ou non, émanant des établissements d'enseignement et de recherche français ou étrangers, des laboratoires publics ou privés. 


\title{
Learning Users' and Personality-Gender Preferences in Close Human-Robot Interaction
}

\author{
Arturo Cruz-Maya and Adriana Tapus \\ Autonomous Systems and Robotics Laboratory, U2IS \\ ENSTA-ParisTech, Université Paris-Saclay, Palaiseau, France \\ Email: \{firstname.lastname\}@ensta-paristech.fr
}

\begin{abstract}
Robots are expected to interact with persons in their everyday activities and should learn the preferences of their users in order to deliver a more natural interaction. Having a memory system that remembers past events and using them to generate an adapted robot's behavior is a useful feature that robots should have. Nevertheless, robots will have to face unknown situations and behave appropriately. We propose the usage of user's personality (introversion/extroversion) to create a model to predict user's preferences so as to be used when there are no past interactions for a certain robot's task. For this, we propose a framework that combines an Emotion System based on the OCC Model with an Episodic-Like Memory System. We did an experiment where a group of participants customized robot's behavior with respect to their preferences (personal distance, gesture amplitude, gesture speed). We tested the obtained model against preset behaviors based on the literature about extroversion preferences on interaction. For this, a different group of participants was recruited. Results shows that our proposed model generated a behavior that was more preferred by the participants than the preset behaviors. Only the group of introvert-female participants did not present any significant difference between the different behaviors.
\end{abstract}

\section{INTRODUCTION}

Robots are expected to interact with persons in their everyday activities, and robots should do it in a way that does not increment the level of stress or anxiety on the users. Therefore, robots should learn the preferences of their users. Having a memory system that remembers past events and using it to predict the outcome of the robot's behavior is a useful feature that robots should possess.

Research in social robotics has shown that robots with emotions and personality are considered to have more believable behavior and are preferred over robots that lack these features [9]. Also, emotions have proved to be important for cognition, and some models have been designed to represent the emotions and their role on cognition. One of them is the OCC model [15], which has been used in virtual agents [2] and in some robots [9] to embody them with emotions and make their behavior more appealing to the users.

The OCC model of emotions lacks the specification of the agent's memory and how the agent's behaviors are modulated with the use of emotions. The memory system combined with emotions has been adopted in [10], where an Episodiclike Memory System (EM) with emotions regulating the intensities of memories on a robot is used. The EM has the advantage of learning online through experience and the capacity to predict expected events based on past experiences.
Here, we propose the usage of the OCC model to synthesize the emotion that regulates the intensities of the memories saved on the EM.

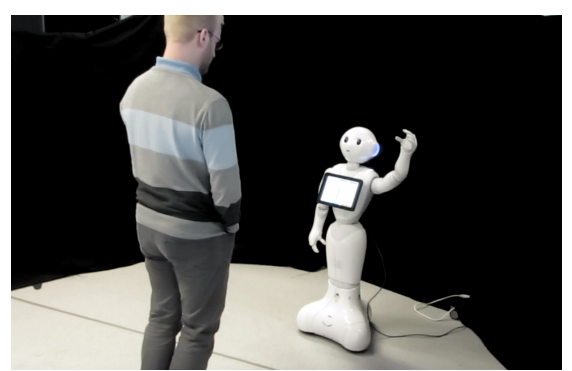

Fig. 1: Interaction scenario with a Pepper Robot

Combining the OCC Model with an Episodic Memory system can give to the robots the capability of being more believable to the users, showing behaviors more preferred by their users. These behaviors should be learned through an interaction between the robots and the users. This information can also be used to interact with new users, if for example the robot knows a priori the personality of a new user, then the robot could deliver an appropriate behavior for the type of personality of this new user.

In the field of Social Psychology, it is widely known that individuals with the same personality have similar preferences. Nevertheless, it exists two theories that seem opposite of each other. One of them is called the similarity attraction theory [3], which states that people are attracted by other people with similar personality. The second one is called complementary needs theory [20], which states that people are attracted by other people with different personality. Both theories have been supported by works in social robotics. In [11], participants enjoyed interacting with an AIBO robot when the robot's personality was complementary to their own preferences. In [7], similarity attraction theory was supported when the robot was a tour guide, and the complementary theory was supported when the robot was a cleaner.

Using an adaptive behavior could be useful to predict the preferences of individuals, when there are no past interactions between them and the robot, if the robot has interacted with other people in similar situations. Instead of having assumptions on any of these theories, the robot would create its own model of user's preferences based on previous experiences. This can be used to model different 
preferences for different tasks. In this work, we describe our proposed framework for an adaptable robot behavior, as well as an experiment divided in two parts. In the first part, the model of the robot's behavior is created. A group of participants interacts with the robot three times, in different days each time, and they specify their preferences for three parameters defining the robot's behavior in a close interaction (as shown in Fig. 1). The preferences are saved individually for each user and also grouped by personality and gender. In the second part of the experiment, the current state of the robot's memory is used to generate the saved behavior to other group of participants with respect to their personality and gender.

There exist many works where a robot is trained from several demonstrations and learn to generate the desired behavior [1]. However, there are only a few works with robots learning online, through interactions with their users, which could be a better form of learning due to the constantly changing nature of the human's preferences.

In a previous work, an assistive therapist robot that adjusted its behavior parameters with respect to the personality and performance of the user was presented [17]. A humanoid torso robot mounted on a mobile platform was used with an algorithm based on a policy gradient reinforcement learning for the adaptation. Personalizing a robot by learning the preferences of their users has been approached before in [14]. The authors developed a robotic system that interacts with the user, and through repeated interactions, adapts to the user. They used a beta regression classifier for learning and generalization of the task, they tested their system with a "room cleaning" scenario in a simplified world, where a small robot with a holonomic base was navigating and transporting blocks to the desired position of the users.

A recent work on engagement and its relation to personality in human robot interaction, is presented in [16]. They propose an automatic analysis of users' personality in a triadic human-human-robot interaction. The analysis is used to predict the personality of the users, and this information is used as an input for the engagement classification system. They focus on the concept of "group engagement" and found better engagement when the participants and the robot were extroverted, and the worst results were obtained when all were introverted.

Using information about the interaction of the robots with the users so as to personalize the interaction over time, allows for more successful and natural human-robot interactions [12]. We propose a framework that includes an Adapted Episodic Memory-like system, which is extended with an Emotion system based on the OCC Model to update the intensity of the memories of past interactions with respect to the user's feedback. This framework has not only to remember the preferences of specific users, but also to generate behaviors for new users based on their personalities. The difference of our proposed model and other existing research works, is that it can generate a specific behavior for each user based on previous interactions, and also, it can generate personality-based behaviors, using the interactions with different users instead of preset information.

This work is structured as follows: section II presents the methods used, section III describes the experimental design setup, results and discussion are showed in section IV, and finally the conclusion is presented in section V.

\section{METHODS}

The high level framework used here was introduced in a previous work [4], where the OCC Model was used to generate predefined behaviors based on the performance of the user. In this work, the model of emotions is used to regulate the intensities of the memories saved on the EpisodicMemory Like, based on past interactions and new user's feedback. The purpose of the framework is to remember users' preferences with respect to a certain robot's behavior that is saved in the Episodic-like Memory (EM). This allows the memories to be in constant variability of recall, more frequent events have a higher recallability. The intensity with which these events are saved depends on the users' feedback and the emotion generated by the Emotion System. We assume that the robot has a priori information about the user, including the gender and personality of the user. The three parameters defining robot's behavior that users can change are: personal distance, gesture amplitude, and gesture speed, having 5 possible values for each parameter.

The process of the proposed algorithm is showed in Fig. 2. It is started by a search of the user's preferences for a specific behavior of the robot. If there exist information about this in the EM, then the behavior is performed with the preferences of the user, if there is no information about the user's preferences, then a search based on the personality and the gender of the user is done. The behavior based on this information, if there is any, is performed, otherwise a default behavior is executed. The user can modify the behavior of the robot by changing the parameters defining robot's behavior (i.e., personal distance, gesture amplitude, gesture speed) and this is done by voice command. After the user finished with the modifications, the parameters are updated with a new weight, rating them with respect to the new user's preferred parameters. The OCC model is used to calculate this weight using the emotion of Hope, which according to this model, is an emotion belonging to the category Consequences of Events having a prospect relevant.

\section{A. Adapted Episodic-like Memory}

The AEM-ART model [18] is made of three layers: Input, Events, and Episodes. Our framework uses the AEMART implementation presented in [10]. The input layer is categorized by channels, each channel containing different elements that are called categories. Also, each category has a channel relevance and activation value, the relevance allows the system to save general information in that channel when its value is 0 , and save specific information when its value is 1. The activation value is used to match a search depending on the channel relevance.

We are saving the information in two types of episodes, one of them is used to save the specific preferences of the 


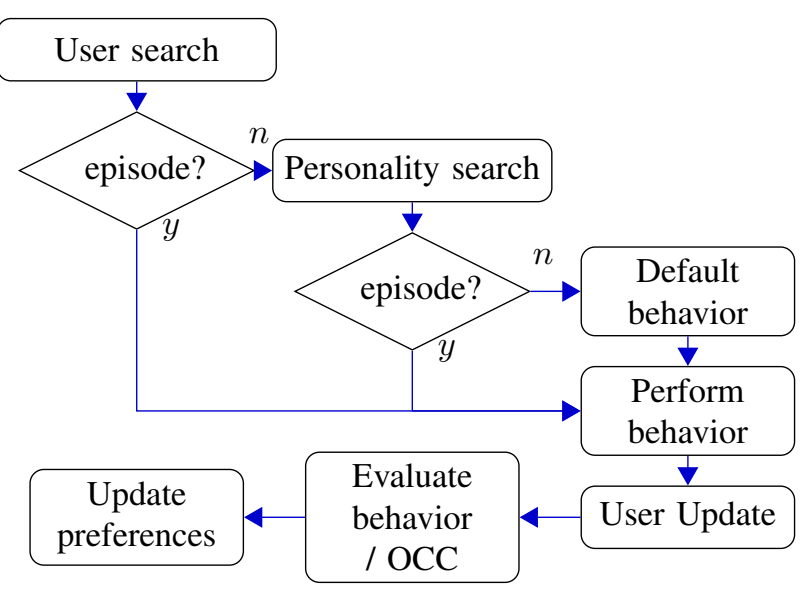

Fig. 2: Process of the proposed framework

users, and the other one is used to save the preferences grouped by personality type (extroversion/introversion) and gender (male/female).

1) Episodes with specific information: For the episodes containing specific user's preferences we used the following channels in the input layer:

- User: Category = Name of the user (unique), channel relevance $=1.0(\max )$, activation value $=1.0(\max )$.

- Order: Used to facilitate the memory search, Category = 1: search, 2: saved preferences, channel relevance $=$ 1.0 , activation value $=1.0$

- Distance: Preferred distance between the robot and the user, given in meters, Category $=[0.4,0.6,0.8,1.0,1.2]$, channel relevance $=1.0$, activation value $=1.0$

- Amplitude: Preferred amplitude of the gesture of the robot's arm, given in percentage of the total amplitude, Category $=[0.2,0.4,0.6,0.8,1.0]$, channel relevance $=$ 1.0 , activation value $=1.0$

- Speed: Preferred speed of the gesture of the robot's arm, given in percentage of the total speed, Category $=$ $[0.2,0.4,0.6,0.8,1.0]$, channel relevance $=1.0$, activation value $=1.0$

- Emotion: Emotion of Hope generated using the OCC Model, Category $=$ 'hope', channel relevance $=1.0$ (max), activation value between 0 and 1 .

The search is done with the channels of the User, Order, and Emotion. The Emotion is used to decrease the importance of past preferences (explained in Section II-B). In this work, we setup the memory to retrieve always the last Episode of the user (Emotion at max. value), meaning his/her last preferences. This gives us episodes with two events, where the first event is used for the search, and the second event contains all the channels described above. A new episode is created for each new user or new preferences of a known user.

2) Episodes with personality/gender information: For the episodes containing information grouped by personality and gender, we used the following channels in the input layer:

- Personality: Category $=$ Personality of the user (Extrovert/Introvert $)$, channel relevance $=1.0(\max )$, activation value $=1.0(\max )$

- Gender: Category $=$ Gender of the user (Male/Female), channel relevance $=1.0$, activation value $=1.0$

- Order: Used to facilitate the memory search, Category = 1: search, 2: saved preferences, channel relevance $=$ 1.0 , activation value $=1.0$

- Distance: Preferred distance between the robot and the user, given in meters, Category $=[0.4,0.6,0.8,1.0,1.2]$, channel relevance $=0.0$, activation value between 0 and 1 , generated using the emotion system.

- Amplitude: Preferred amplitude of the gesture of the robot's arm, in percentage of the total amplitude, Category $=[0.2,0.4,0.6,0.8,1.0]$, channel relevance $=0.0$, activation value $=$ between 0 and 1 , generated using the emotion system.

- Speed: Preferred speed of the gesture of the robot's arm, in percentage of the total speed, Category = $[0.2,0.4,0.6,0.8,1.0]$, channel relevance $=0.0$, activation value $=$ between 0 and 1 , generated using the emotion system.

The episodes generated using this channel configuration contain two events, one for the memory search (Personality, Gender, Order) and the other one with all the channels presented above. The channels of Distance, Amplitude, and Speed (parameters' channels) are set up with a channel relevance of 0 in order to save general information on them, grouped by personality and gender, which were set up with a channel relevance of 1 . The activation value of each Category in the parameters' channels are updated each time a user save his/her preferences, increasing the activation of preferred parameters with a value in an empirically calculated range from 0 to 0.2 . This value depends on the emotion generated by the emotion system. The activation of each Category of non preferred parameter is decreased by a factor of $5 \%$ of its current value. This is done with the purpose of slowly forgetting not frequently chosen parameters.

\section{B. OCC Model}

The OCC Model [15] is a model commonly used in virtual agents to synthesize emotions, it has 22 emotions and they are divided in three categories: Aspect of Objects, Action of Agents, and Consequences of Events. In this work, we focused on the category of Consequences of Events for Self, especially in the emotion of Hope. This can improve the Adapted-Episodic-like Memory system rating the memories with higher activation values for events more liked by the users. We use the Hope emotion because it is a prospect based emotion, unlike emotions of joy or distress which are well-being emotions having prospects irrelevant or others emotions which are based on Consequences for Others.

We have two types of episodes, one for specific user's preferences and one for personality/gender preferences. We generate the emotion of Hope for each type of episodes.

1) Emotion for episodes with specific information: For the type of episodes with information about specific user's preferences, each time a search is done in the memory system, an emotion of Hope is generated. We used 4 global 
variables of the OCC model and 2 local variables, all of them normalized with values between 0 and 1 .

The global variables are:

- Sense of Reality $(S o R)$ : value $=0.75$, because this emotion is based on the memories of a specific user.

- Proximity (Prox): value $=1$, because our scenario presents a close interaction between the robot and the user,

- Unexpectedness $(U n)$ : value $=1-$ EpisodeActivation (memory system)

- Arousal $(A r)$ : value $=0.5$, in order to set a neutral value for this variable.

The global variables were combined computing the mean of all of them (Eq. 1).

$$
\text { GlobalVar }=(S o R+\operatorname{Prox}+U n+A r) / 4
$$

The values of the local variables (Likelihood and Desirability) are computed as follows:

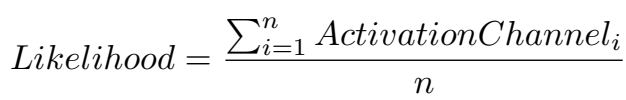

where $\mathrm{n}$ is the number of parameters' channels (three in this case)

$$
\text { Desirability }=1
$$

The value of Desirability was set to 1 , because there is only one objective in the task performed by the robot.

In the OCC model, the potential of the emotion is obtained by combining the global variables with the local variables. Each emotion has also a threshold to discard emotions with low potential. In this work, the emotion potential is computed as shown in Eq. 4. The threshold of the emotion was set to 0 to allow an intensity of the emotion ranging from 0 to 1 .

$$
E m P=(\text { Des } * 0.7+\text { GlobalVar } * 0.3) * L k
$$

where $E m P$ is the Emotion Potential, Des is the Desirability, GlobalVar are calculated using the Equation 1, and $L k$ is the Likelihood.

After the user gives the feedback to the robot, there are two possible emotions that are consequence of the emotion of Hope. These emotions are Satisfaction Confirmed and Disappointment. Satisfaction confirmed happens if the user's preferences remain unchanged. Then, the synthesized emotion of Hope is assigned to the preferred parameter. Disappointment happens if the user's preferences are different than the remembered preferences. Then, the emotion assigned to the preferred parameter is $1-$ Hope. The final emotion is the average of all the emotions of each parameter, which is used as an activation value in the category of Hope in the emotion channel of the memory system. New events, which are the preferences of the user, are set with a high level of emotion in order to be easy remembered.
2) Emotion for episodes with personality/gender information: For the type of episodes with information about personality/gender, we used the same variables, changing the Sense of reality to 0.5 , because the memory retrieved is composed by several users. For the same reason, the values of the Desirability and Likelihood need to be computed based on the information in the memory system, as well as a prediction of the preferred parameters.

The predicted parameters are computed by using the information contained in the Episodic-like Memory as follows:

$$
\operatorname{Param}_{k}=\frac{\sum_{i=1}^{n} C h_{k}\left[C a t_{i}\right] * C h_{k}\left[A c t_{i}\right]}{\sum_{i=1}^{n} C h_{k}\left[A c t_{i}\right]}
$$

where $k$ is the channel of the predicted parameter, Cat $_{i}$ is the Category $i$ of the channel $k$, and $A c t_{i}$ is the activation $i$ of the channel $k$. Each Param $_{k}$ is rounded to a value multiple of 0.2 in order to discretize them. When there are no preferences saved in memory, the predicted value is set to 0.6. Param $_{k}$ is associated with an activation value $\operatorname{Param}_{k}[$ Act $]$, corresponding with the category with the same value of the channel $k$.

The likelihood of each predicted parameter is:

$$
l k_{k}=\frac{\operatorname{Param}_{k}[\text { Act }]}{\sum_{i=1}^{n} C h_{k}\left[\text { Act }_{i}\right]}
$$

where $k$ is the channel of the predicted parameter, and $n$ is the number of categories in the channel $k$ and $A c t_{i}$ is the Activation $i$ of the channel $k$.

The local variables were computed as follows:

$$
\begin{gathered}
\text { Likelihood }=\frac{\sum_{k=1}^{n} l k_{k}}{n} \\
\text { Desirability }=\frac{\sum_{i=1}^{n} C h_{i}[M a x(\text { Act })]}{n}
\end{gathered}
$$

where $n$ is the number of parameter channels.

The emotion is computed as described above.

\section{EXPERIMENTAL DESIGN SETUP}

\section{A. Hypothesis}

Remembering user's preferences is one of the basic capacities that personal robots should have, but these preferences should not be fixed over time. Robots should be able to change these parameters with respect to the preferences of the users. The inter-individual differences based on personality and gender need to be integrated and the robot's behaviors should best match the preferences of new users.

Our hypothesis is stated as follows:

H1. The robot's behavior generated after interactions with individuals of different personalities and genders will best match with the preferences of new users than by using fixed behaviors based on the theories of Similarity attraction or Complementarity attraction. 


\section{B. Scenario Description}

The purpose of this work was to customize a robot's behavior to users' preferences. This learned behavior was shown to new users, using the information saved in the Episodic Memory to perform the behavior with the most common preferred parameters by individuals with the same gender and personality. For this reason, we did an experiment divided in two parts. The first part was done to test the Episodic-like Memory (EM) and the behavior of the robot, and to train a model of users' preferences based on personality and gender. The second part was done in order to test the models created by the EM. The behavior of the robot was expressed through three parameters configured by the user: personal distance, gesture amplitude, and gesture speed. We chose these parameters because of the their link and corelation with the extroversion/introversion personality trait. It is well known that extroverted individuals tend to do wider and faster gestures[8] [13], and also prefer closer distances than introverted individuals [19]. The gesture used was a wave of the left arm of the robot, when the robot was greeting the user.

\section{Robot Capabilities}

The robot used was a Pepper robot, which has the capabilities of Face Detection and Face Tracking, Voice Recognition, and movement of the body and head to track the user. These capabilities are provided by the NaoQI Framework. We used the naoqi ROS driver to communicate with the Episodiclike Memory and Emotion systems that were located on an external computer.

\section{Training the robot}

In order to train our model based on personality and gender, 16 Participants were recruited to perform this experiment, 8 male and 8 female participants, with ages between 25 and 56 years old.

The experiment was done as follows. The participants were instructed to interact one by one with the robot in a dyadic interaction, the robot was able to recognize a list of voice commands (see below). Participants were standing in front of the robot. The robot greeted the participant with a hand wave and verbal greeting, "Hello, I'm learning about personalities preferences for which I will interact with you for some minutes. You can modify three parameters of my behavior: personal distance, gesture's amplitude and gesture's speed". The robot listed the voice commands and showed them on the tablet mounted on its torso (see Fig.3). Then the participants were free to give the desired commands to modify the parameters of the robot. It was repeated 3 times for each participant in different days each time.

The voice commands and their purpose are as follows: (a) Show Commands: To show on the tablet the list of voice commands; (b) Modify distance: To modify the distance between the robot and the user; (c) Modify gesture: To modify the gesture's amplitude; (d) Modify Speed: To modify the gesture's speed; (e) Reduce: To reduce the chosen parameter; (f) Increase: To increase the chosen parameter; (g) Done: (a)

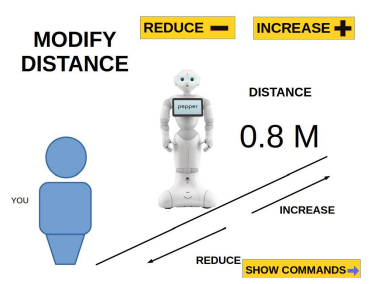

(c)

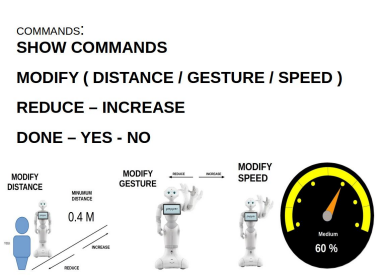

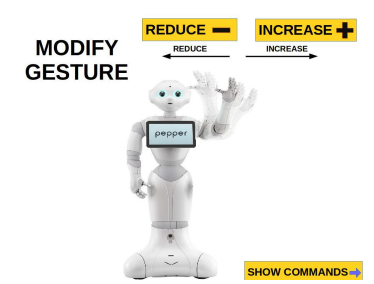

(b)

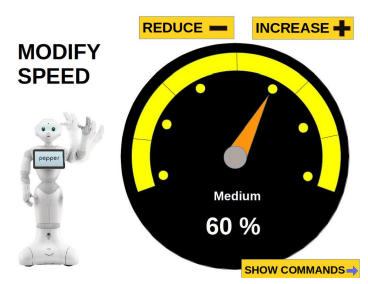

(d)
Fig. 3: Images showed on the tablet of the robot. a) Image to show the voice commands, b) c) d) Images displayed when the user was modifying each parameter.

TABLE I: Amplitude of the wave of the robot's arm

\begin{tabular}{ll|l}
\hline & Minimum & Maximum \\
\hline Shoulder Roll & $40.1^{\circ}$ to $42.4^{\circ}$ & $40.1^{\circ}$ to $51.5^{\circ}$ \\
Shoulder Pitch & $68.75^{\circ}$ & $68.75^{\circ}$ \\
Elbow Roll & $-74.5^{\circ}$ to $-51.5^{\circ}$ & $-74.5^{\circ}$ to $-5.7^{\circ}$ \\
Elbow Yaw & $0^{\circ}$ & $0^{\circ}$ \\
Wrist Yaw & $34.4^{\circ}$ & $34.4^{\circ}$ \\
\hline
\end{tabular}

To finish the modifications; (h) Yes: To confirm the "Done" command; (i) No: To cancel the "Done" command. Each time the user said one of the commands to modify a parameter, one image was illustrated on the torso tablet that showed the robot and the current value of the parameter in terms of percentages for the speed and amplitude, and in meters for the distance. The used images are shown in Fig. 3. The distance had values in a range of $40 \mathrm{~cm}$ to $120 \mathrm{~cm}$ (as stated by Hall [6]). The minimum and maximum values for the amplitude of the wave of the robot's arm are shown in Table I, a full schema of the joints and angles is shown in the documentation website ${ }^{1}$ of the robot. The speed had values between $40 \%$ to $100 \%$ of the maximum speed provided by SoftBank Robotics, details of the motor of each joint are shown in the documentation website ${ }^{2}$ of the robot. The robot performed the behavior each time the user said "increase" or "decrease" the chosen parameter. Learning is done after the first interaction for each user of each personality/gender group.

\section{E. Pre-experiment Questionnaire}

The participants were chosen with respect to their scores on a personality test (Big 5 [5]). The personality test was filled-in by the participants before the experiments. Individuals with a score $<=3$ on a personality trait were considered

\footnotetext{
${ }^{1}$ http://doc.aldebaran.com/2-0/family/juliette_technical/joints_juliette.html

${ }^{2}$ http://doc.aldebaran.com/2-0/family/juliette_technical/motors_juliette.html
} 
in the low category of that specific personality trait. The training phase was done with 16 participants: 4 in each group (introverted/extroverted and male/female). The testing phase was done with 26 participants: 8 introverted males, 9 extroverted males, 4 introverted females, and 5 extroverted females.

\section{F. Post-Experiment Questionnaire - Training Phase}

A post-experiment questionnaire (10 items) was applied to the participants that modified the parameters of the robot's behavior. The first 6 questions were designed to know if individuals preferred lower or higher values for the min and max parameters, and the other 4 questions were designed to know if individuals considered the parameters they chosen as appropriate, with answers on a 5 points Likert scale. Most of the participants did not like lower minimum values or higher values for the parameters of the robot's behavior. The percentages of the participants for the answers of the questions 1 to 6 are shown in Table II. Also, most of the participants rated the chosen parameters as appropriate (4 on a scale from 1 to 5 ) in the questions 7 to 10 (as shown in Table III). The final behavior was most of the times rated as neutral.

\section{G. Testing of the model}

In order to test our model based on personality and gender, 26 Participants were recruited to perform this experiment, 17 male and 9 female participants, with ages ranging from 20 to 47 years old. Participants taking part in the training phase were not part of the testing group. We designed two robot behaviors based on the literature about introverts and extroverts, the values of our interaction parameters were chosen using the minimum and maximum values chosen by the participants in the training phase. The values of each parameter for each personality of the robot are shown in Table IV.

TABLE II: Answers of participants in the Training phase Questions 1-6

\begin{tabular}{lrr}
\hline & Yes & No \\
\hline Lower Distance & $6.2 \%$ & $93.8 \%$ \\
Higher Distance & $37.5 \%$ & $62.5 \%$ \\
Lower Amplitude & $25.0 \%$ & $75.0 \%$ \\
Higher Amplitude & $18.8 \%$ & $81.2 \%$ \\
Lower Speed & $6.2 \%$ & $93.8 \%$ \\
Higher Speed & $43.8 \%$ & $56.2 \%$ \\
\hline
\end{tabular}

TABLE III: Answers of participants in the Training phase Questions 7-10, Likert Scale: 1 (not at all appropiate) to 5 (very appropriate)

\begin{tabular}{llllll}
\hline & 1 & 2 & 3 & 4 & 5 \\
\hline Chosen Distance & $6.25 \%$ & $12.5 \%$ & $0.00 \%$ & $75.0 \%$ & $6.25 \%$ \\
Chosen Amplitude & $0.00 \%$ & $0.00 \%$ & $31.3 \%$ & $50.0 \%$ & $18.7 \%$ \\
Chosen Speed & $0.00 \%$ & $18.7 \%$ & $25.0 \%$ & $43.8 \%$ & $12.5 \%$ \\
Final Behavior & $06.25 \%$ & $0.00 \%$ & $56.2 \%$ & $37.5 \%$ & $0.00 \%$ \\
\hline
\end{tabular}

TABLE IV: Parameters for the introvert and the extrovert robot's behavior

\begin{tabular}{llll}
\hline & Distance (m) & Amplitude (\%) & Speed (\%) \\
\hline Extro Robot & 1.20 & 100.0 & 100.0 \\
Intro Robot & 0.40 & 20.0 & 40.0 \\
\hline
\end{tabular}

Each participant observed the robot performing the behavior generated by our model with respect to his/her personality and gender, the introverted behavior of the robot, and the extroverted behavior of the robot. The robot's behaviors were presented in a random order to each participant. At the end of each robot's behavior, the participant completed a questionnaire with three questions about his/her preferences about the three parameters to evaluate (distance/amplitude/speed). The answers were on a 5 points Likert scale, where 1 was "too close/narrow/slow" for the distance/amplitude/speed respectively, "3" was "appropriate", and 5 was "too far/wide/fast", respectively. Other three questions were used to rate the perceived stress caused by each parameter of robot on a 5 points Likert scale.

\section{RESULTS AND DISCUSSION}

\section{A. Training the robot}

The values for each parameter, obtained at the end of the training of the robot are shown in Fig. 4, and in Table $\mathrm{V}$, where the values in parenthesis are the values that the robot can perform, and that are the actual values that were used in the second part of the experiment. The model predicts that extroverted female participants will prefer a closer distance than the other groups of participants, also that female participants will prefer gestures with higher amplitude than male participants with the same personality as theirs (extroversion/introversion) and that extroverted male participants will prefer a faster speed for the gestures than the other groups of participants. As our model is based on a weighted mean of the activation values of each parameter in the EM, we applied a weighted t-test using the software R for a statistical analysis on the differences of the predicted parameters. The only significant difference was found in the preferred amplitude between the extrovert female group $(0.83$ $\mathrm{m})$ and the introvert male group $(0.44 \mathrm{~m})$ with $t=2.327$, $d f=7.905, p=0.048$, and Std.err $=0.167$.

\section{B. Testing the model}

We compared our model against two different behaviors of the robot based on the introversion and extroversion person-

TABLE V: Predicted parameters of the robot's behavior by personality and gender

\begin{tabular}{llll}
\hline & Distance $(\mathrm{m})$ & Amplitude $(\%)$ & Speed $(\%)$ \\
\hline Extro Female & $0.69(0.60)$ & $83.0(80.0)$ & $86.0(80.0)$ \\
Extro Male & $0.73(0.80)$ & $66.0(60.0)$ & $93.0(100.0)$ \\
Intro Female & $0.82(0.80)$ & $63.0(60.0)$ & $74.0(80.0)$ \\
Intro Male & $0.87(0.80)$ & $44.0(40.0)$ & $70.0(60.0)$ \\
\hline
\end{tabular}




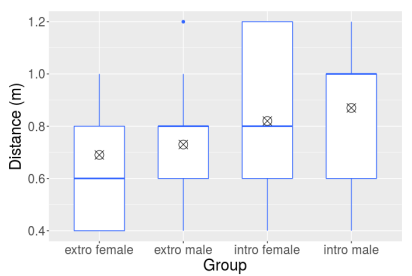

(a)

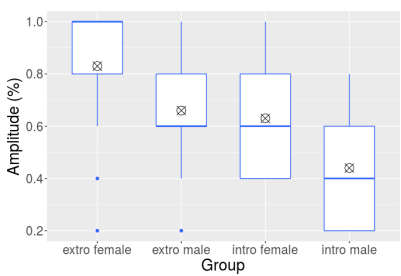

(b)

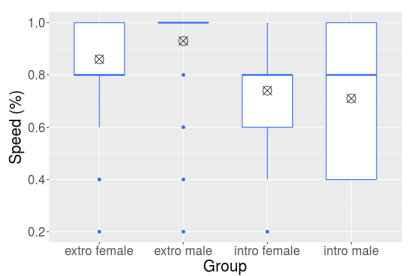

(c)

Fig. 4: Predicted parameters of gesture by Personality/Gender. a) distance b) Amplitud c) Speed.

ality traits. The means of the answers of the participants for each behavior on a 5 points Likert scale are shown in Table VI. We obtained different results for each personality-gender group. For each parameter (distance/speed/gesture) defining the behavior of the robot a two-way ANOVA was performed to identify main effects or interaction effects between the parameters and the personality and gender of the participants. We did not found any significant effect. A possible cause of this is the small number of participants that took part in the experiment. For this reason, we divided the data by personality/gender and analyzed it separately with a pairwise t-test for each group.

1) Extrovert-Male: The distance of the proposed model was perceived as "more appropriate" than the distance of the extroverted robot behavior ( $p=0.0087$ ). The amplitude of the introverted behavior was perceived as "more appropriate" than the amplitude of the extroverted behavior $(p=0.02)$. In Table VI, Extro $M$ section shows the means of each parameter of the different behaviors of the robot. The model has a mean closer to 3 ("appropriate") than the introverted and extroverted behaviors in the parameters of distance and amplitude. This can also be seen in Figs. 5b and 5c. The speed of the extroverted robot was chosen as "appropriate" more times than the other behaviors (see Fig. 5a).

2) Introvert-Male: The distance of the introverted robot behavior was perceived as "less appropriate" than the distance of the proposed model $(p=0.0022)$, and as "more appropriate" than the extroverted robot behavior (0.0014). In Table VI, Intro $M$ section shows the means of each parameter of the different behaviors of the robot. The model has a mean closer to 3 ("appropriate") than the introverted and extroverted behaviors in the parameters of distance and speed.

3) Extrovert-Female: The distance of the introverted robot behavior was perceived as "more appropriate" than the distance of the proposed model $(p=0.0269)$, and than the
TABLE VI: Comparison of users' preferences of the generated model and the introvert and the extrovert robot's behaviors. Means and Variance of the 5 points Likert scale questionnaire.

\begin{tabular}{llll}
\hline Extro M & Model & Intro R & Extro R \\
\hline Speed & $3.55(0.77)$ & $2.66(2.25)$ & $\mathbf{3 . 2 2}(\mathbf{0 . 1 9})$ \\
Amplitude & $\mathbf{3 . 2 2}(\mathbf{0 . 1 9})$ & $2.66(1.25)$ & $3.55(0.27)$ \\
Distance & $\mathbf{2 . 7 7}(\mathbf{0 . 4 4})$ & $3.33(0.50)$ & $1.88(0.36)$ \\
\hline Intro M & Model & Intro R & Extro R \\
\hline Speed & $\mathbf{2 . 8 7}(\mathbf{0 . 6 9})$ & $2.75(0.50)$ & $3.25(0.50)$ \\
Amplitude & $2.75(0.78)$ & $\mathbf{3 . 0 0}(\mathbf{0 . 8 5})$ & $3.50(0.57)$ \\
Distance & $\mathbf{3 . 0 0}(\mathbf{0 . 2 8})$ & $3.37(0.83)$ & $1.87(0.12)$ \\
\hline Extro F & Model & Intro R & Extro R \\
\hline Speed & $\mathbf{3 . 0 0}(\mathbf{0 . 5})$ & $2.40(0.8)$ & $3.80(0.2)$ \\
Amplitude & $\mathbf{3 . 6 0}(\mathbf{0 . 3 0})$ & $3.00(1.00)$ & $2.80(1.20)$ \\
Distance & $2.60(1.30)$ & $\mathbf{3 . 3 7}(\mathbf{0 . 8 3})$ & $1.80(0.20)$ \\
\hline Intro F & Model & Intro R & Extro R \\
\hline Speed & $2.50(0.33)$ & $2.50(0.33)$ & $3.25(0.25)$ \\
Amplitude & $\mathbf{3 . 2 5}(\mathbf{0 . 2 5})$ & $2.75(0.91)$ & $3.50(0.33)$ \\
Distance & $3.25(0.25)$ & $3.75(0.25)$ & $2.75(0.25)$ \\
\hline
\end{tabular}

extroverted robot behavior (0.0012). The speed of the introverted behavior was perceived different as "somewhat far", while the extroverted behavior was perceived as "somewhat close / very close" ( $p=0.0087)$. In Table VI, Extro F section shows the means of each parameter of the different behaviors of the robot, the model has a mean closer to 3 ("appropriate") than the introverted and extroverted behaviors in the parameter of speed.

4) Introvert-Female: There were no significant differences on the parameters of the different robot behaviors. In Table VI, Intro F section shows the means of each parameter of the different behaviors of the robot.

The behavior of the robot generated by the proposed model got an advantage over the introverted behavior and the extroverted behavior in some parameters in most of the personality/gender groups. Even when the differences were not significantly different, we can see a better performance of it. A larger group of participants would be needed so as to verify the differences. The parameter of distance of the proposed model was rated as "appropriate" more than the distance of the other behaviors with a difference that showed to be statistically significant as stated above. The accuracy of the model can be improved by training with more users. Also, for the sake of simplicity, the robot's behavior was restricted to 5 values for each parameter. Otherwise, the model could had be better rated by the participants in the testing phase. We can see that introverted male participants rated with a mean of 2.87 the speed of the robot's gesture generated with the model (see Table VI), meaning something slower than an appropriate value, the used value was $60 \%$, but the value given by the model was $70 \%$, which was a higher speed. Similar to this, the extroverted participants rated the speed of the robot's gesture generated with the model as faster than an appropriate value (mean 3.55), the used value was $100 \%$, but the value given by the model was $93 \%$, which was a slower speed. This shows that personality and gender can be used to predict user's preferences of 


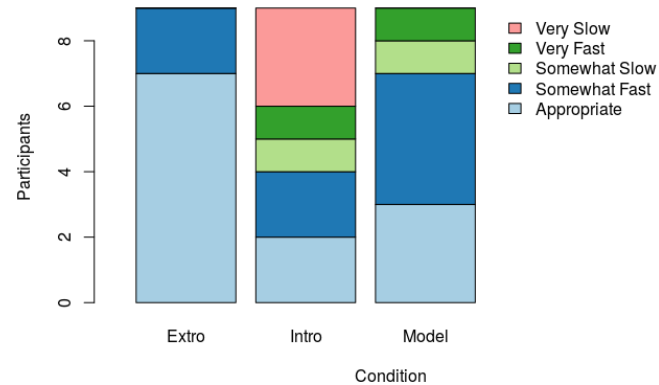

(a)

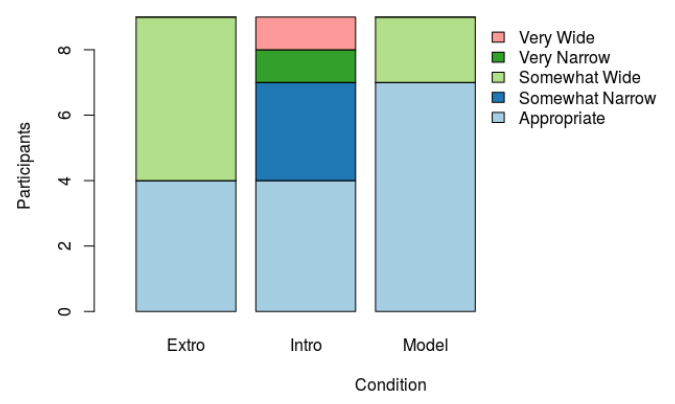

(b)

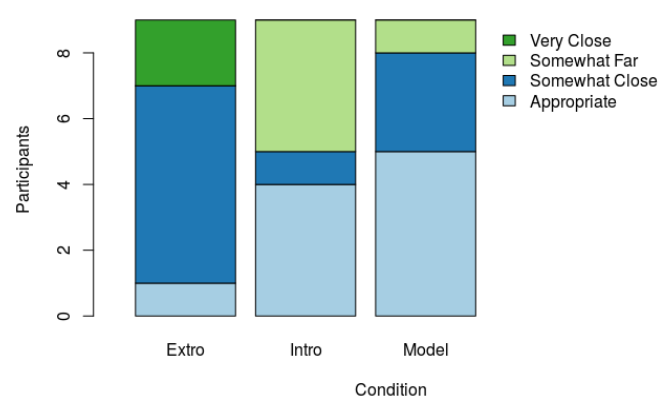

(c)

Fig. 5: Parameter preferences of the Extrovert-Male participants in the different robot behaviors. Extro: Extroverted robot, Intro: Introverted robot, Model: Proposed Model. a) Speed b) Amplitud c) Distance

robot's behaviors. Our hypothesis, that the robot's behavior generated with the interaction of individuals of different personalities and gender will best match with preferences of new users, was partially confirmed, as our model did not fit all the parameters of the different groups. Nevertheless, we are convinced that if we train our model with more individuals it can give better results.

\section{CONCLUSION AND FUTURE WORK}

In this work, we presented a framework based on an Episodic Memory-Like and a cognitive model of emotions (OCC) to create a robot model behavior based on the personality and gender of its users. We trained the model with 16 participants, 4 participants for each group of personality/gender, and tested it with other 26 participants. Results are promising as per the discussions above. Future works include the use of different behaviors occurring in a consecutive time and different contexts, taking advantage of the episodic memory and the proposed model.

\section{ACKNOWLEDGMENT}

The authors thank to the Mexican Council of Science and Technology for the grant CONACYT-French Government n.382035. This work was also funded by EU Horizon 2020 ENRICHME project grant agreement no. 643691C.

\section{REFERENCES}

[1] B. D. Argall, S. Chernova, M. Veloso, and B. Browning, "A survey of robot learning from demonstration," Robotics and autonomous systems, vol. 57, no. 5, pp. 469-483, 2009.

[2] C. Becker-Asano and I. Wachsmuth, "Affective computing with primary and secondary emotions in a virtual human," Autonomous Agents and Multi-Agent Systems, vol. 20, no. 1, p. 32, 2010.

[3] D. Byrne, W. Griffitt, and D. Stefaniak, "Attraction and similarity of personality characteristics." APA Journal of Personality and social Psychology, vol. 5, no. 1, p. 82, 1967.

[4] A. Cruz-Maya, F. Ferland, and A. Tapus, "Social facilitation in a gamelike human-robot interaction using synthesized emotions and episodic memory," in International Conference on Social Robotics. Springer, 2015, pp. 164-173.

[5] L. R. Goldberg, "An alternative 'description of personality': the bigfive factor structure." APA Journal of Personality and Social Psychology, vol. 59, no. 6, 1990.

[6] E. T. Hall, The hidden dimension. Doubleday \& Co, 1966.

[7] M. Joosse, M. Lohse, J. G. Pérez, and V. Evers, "What you do is who you are: The role of task context in perceived social robot personality," in Robotics and automation (ICRA), 2013 IEEE international conference on. IEEE, 2013, pp. 2134-2139.

[8] M. L. Knapp, J. A. Hall, and T. G. Horgan, Nonverbal communication in human interaction. Cengage Learning, 2013.

[9] B. J. Kröse, J. M. Porta, A. J. van Breemen, K. Crucq, M. Nuttin, and E. Demeester, "Lino, the user-interface robot," in European Symposium on Ambient Intelligence, 2003, pp. 264-274.

[10] F. Leconte, F. Ferland, and F. Michaud, "Design and integration of a spatio-temporal memory with emotional influences to categorize and recall the experiences of an autonomous mobile robot," Autonomous Robots, vol. 40, no. 5, pp. 831-848, 2016.

[11] K. M. Lee, W. Peng, S.-A. Jin, and C. Yan, "Can robots manifest personality?: An empirical test of personality recognition, social responses, and social presence in human-robot interaction," Journal of communication, vol. 56, no. 4, pp. 754-772, 2006.

[12] D. Leyzberg, S. Spaulding, and B. Scassellati, "Personalizing robot tutors to individuals' learning differences," in Proceedings of the 2014 ACM/IEEE international conference on Human-robot interaction. ACM, 2014, pp. 423-430.

[13] R. Lippa, "The nonverbal display and judgment of extraversion, masculinity, femininity, and gender diagnosticity: A lens model analysis," Journal of Research in Personality, vol. 32, no. 1, pp. 80-107, 1998.

[14] M. Mason and M. C. Lopes, "Robot self-initiative and personalization by learning through repeated interactions," in Proceedings of the 6th ACM international conference on HRI, 2011, pp. 433-440.

[15] A. Ortony, G. L. Clore, and A. Collins, The cognitive structure of emotions. Cambridge university press, 1990.

[16] H. Salam, O. Celiktutan, I. Hupont, H. Gunes, and M. Chetouani, "Fully automatic analysis of engagement and its relationship to personality in human-robot interactions," IEEE Access, vol. 5, pp. 705$721,2017$.

[17] A. Tapus, C. Tapus, and M. Matarić, "Long term learning and online robot behavior adaptation for individuals with physical and cognitive impairments," in Field and Service Robotics, 2010, pp. 389-398.

[18] W. Wang, B. Subagdja, A.-H. Tan, and J. A. Starzyk, "Neural modeling of episodic memory: Encoding, retrieval, and forgetting," IEEE transactions on neural networks and learning systems, vol. 23 , no. 10, pp. 1574-1586, 2012.

[19] J. L. Williams, "Personal space and its relation to extraversionintroversion." Canadian Journal of Behavioural Science/Revue canadienne des sciences du comportement, vol. 3, no. 2, p. 156, 1971.

[20] R. F. Winch, T. Ktsanes, and V. Ktsanes, "The theory of complementary needs in mate-selection: An analytic and descriptive study," American Sociological Review, vol. 19, no. 3, pp. 241-249, 1954. 